\title{
Uusioersily
}

\section{The role of locus of control and coping style in predicting longitudinal PTSD- trajectories after combat exposure}

Karstoft, K-I., Armour, C., Elklit, A., \& Solomon, Z. (2015). The role of locus of control and coping style in predicting longitudinal PTSD-trajectories after combat exposure. Journal of Anxiety Disorders, 32, 89-94. https://doi.org/10.1016/j.janxdis.2015.03.007

Link to publication record in Ulster University Research Portal

\section{Published in:}

Journal of Anxiety Disorders

Publication Status:

Published (in print/issue): 31/05/2015

DOI:

10.1016/j.janxdis.2015.03.007

\section{Document Version}

Author Accepted version

\section{General rights}

Copyright for the publications made accessible via Ulster University's Research Portal is retained by the author(s) and / or other copyright owners and it is a condition of accessing these publications that users recognise and abide by the legal requirements associated with these rights.

\section{Take down policy}

The Research Portal is Ulster University's institutional repository that provides access to Ulster's research outputs. Every effort has been made to ensure that content in the Research Portal does not infringe any person's rights, or applicable UK laws. If you discover content in the Research Portal that you believe breaches copyright or violates any law, please contact pure-support@ulster.ac.uk. 
The role of locus of control and coping style in predicting longitudinal PTSD-trajectories after combat exposure

\author{
Karen-Inge Karstoft ${ }^{1 *}$; Cherie Armour ${ }^{2}$; Ask Elklit ${ }^{3}$; Zahava Solomon ${ }^{4}$
}

*Corresponding author: Karen-Inge Karstoft, Research and Knowledge Centre, Danish Veteran Centre, Garnisonen 1, 4100 Ringsted, Denmark

Email: kareningekarstoft@gmail.com

Phone: +4572163320

\title{
Author Affiliations
}

${ }^{1}$ Research and Knowledge Centre, Danish Veteran Centre, Ringsted, Denmark

${ }^{2}$ School of Psychology, University of Ulster at Coleraine Campus, Coleraine, Northern Ireland, UK

${ }^{3}$ The National Centre for Psychotraumatology, University of Southern Denmark, Odense, Denmark

${ }^{4}$ Bob Shapell School of Social Work, Tel Aviv University, Tel Aviv, Israel 


\section{Abstract}

While longitudinal posttraumatic stress responses are known to be heterogeneous, little is known about predictors of those responses. We investigated if locus of control (LOC) and coping style are associated with long-term PTSD-trajectories after exposure to combat. Six hundred and seventy five Israeli soldiers with or without combat stress reaction (CSR) from the Lebanon war were assessed one, two, and 20 years after the war. Combat exposure, LOC, and coping style were then investigated as covariates of the trajectories of resilience, recovery, delayed onset, and chronicity. Symptomatic trajectories in the CSR and the non-CSR group were significantly associated to varying degrees with perceived life threat during combat (ORs: 1.76-2.53), internal LOC (0.77-0.87), emotional coping style (0.28-0.34), and low use of problem-focused coping (2.12-3.11). In conclusion, assessment of LOC and coping can aid prediction of chronic PTSD outcomes of combat exposure.

\section{Keywords:}

Trauma, posttraumatic stress disorder, combat, coping, locus of control, veterans 


\section{Introduction}

An unresolved debate in the literature on posttraumatic stress disorder (PTSD) concerns issues of vulnerability to stress-induced psychopathology. Research has indicated that whereas the majority of individuals exposed to traumatic events do not succumb to stress, a significant minority experience subsequent psychopathology (Kessler, Sonnega, Bromet, Hughes, \& Nelson, 1995). Importantly, most of those who develop PTSD remain sensitized and vulnerable to subsequent adversity (Solomon, 1993; Solomon \& Mikulincer, 2006). This study aims to examine the association of two psychological attributes, namely locus of control (LOC) and coping style with long-term PTSD trajectories.

Longitudinal studies investigating trajectories of PTSD in military samples have repeatedly found posttraumatic stress responses to be heterogeneous (Andersen et al., 2014; Bonanno et al., 2012; Dickstein, Suvak, Litz, \& Adler, 2010; Orcutt, Erickson, \& Wolfe, 2004). These studies suggest that while the predominant trajectory is that of resilience, symptomatic trajectories are also observed. More precisely, trajectories of recovery, delayed onset, , and chronicity have most often been identified (Andersen et al., 2014; Bonanno et al., 2012; Dickstein et al., 2010; Orcutt et al., 2004). Since heterogeneous trajectories seem to be the norm, a pertinent aim is the identification of vulnerability markers which will enable the prediction of individual trajectories of stress reactions. This will in turn permit the early identification of individuals who will go on to develop chronic PTSD.

Several variables have been identified as risk factors for PTSD in the general population and in the military. More specifically, demographics (e.g., gender \& age), pre-trauma functioning, peri-traumatic characteristics (such as combat exposure), peri-traumatic functioning 
(e.g. combat stress reaction (CSR); Solomon, 1993), and posttraumatic support all play a role in predicting PTSD (Brewin, Andrews, \& Valentine, 2000; Ozer, Best, Lipsey, \& Weiss, 2003). However, the sum of explained variability in two often cited meta-analyses is low $(<20 \%$; Brewin et al., 2000; Ozer et al., 2003). Thus, a great deal of the variance remains unexplained and additional predictive variables need to be identified. Whereas demographic variables, peritraumatic reactions, and post-traumatic support have often been investigated in studies of military trauma, the implication of psychological attributes in the prediction of long-term PTSDtrajectories has, less often, been under scrutiny.

We aim to examine the role of combat exposure and two psychological attributes in predicting individual differences in long-term post-traumatic stress response in soldiers who did or did not experience a Combat Stress Reaction (CSR). CSR describes acute stress reactions following exposure to PTE in a military context and is defined as a functional breakdown on the battlefield that limits the individual's ability to function as a combatant (Solomon, 1993). More specifically, we will evaluate how locus of control (LOC) and coping style relates to the PTSDtrajectories of chronicity, recovery, resilience, and delayed onset.

\subsection{Locus of control and PTSD}

Locus of control (LOC) concerns the extent to which individuals feel they can control events that affect them (Rotter, 1966). Specifically, it assesses the degree to which individuals believe that events are controllable by their actions (internal LOC) or primarily depend on factors that are beyond their control (external LOC). Research has found that external LOC is related to higher level of PTSD-symptoms (e.g.Casella \& Motta, 1990; McKeever, McWhirter, \& Huff, 
2006; Norris et al., 2002; Solomon, Mikulincer, \& Avitzur, 1988, Zhang et al., 2014). Hence, internal LOC is expected to be a protective factor against PTSD, while an external LOC is a risk factor for the development of PTSD after exposure to combat. Less, however, is known about the general relationship between LOC and long-term PTSD outcome, and whether or not LOC predicts the persistence or chronicity of PTSD (Olff, Langeland, \& Gersons, 2005). Prospective investigation of the predictive qualities of LOC may therefore yield potentially important results, and yet to the best of our knowledge, this has not been investigated in relation to heterogeneous, long-term trajectories of PTSD following combat.

\subsection{Coping style and PTSD}

Coping style is defined as the cognitive and behavioral efforts applied by an individual to manage internal or external demands (Lazarus \& Folkman, 1984). A number of different coping strategies have been conceived and described (e.g., active, passive, avoidant, emotional etc.), and as a result, coping is viewed as a multi-dimensional concept. Indeed, a well-supported coping typology was conceived by Lazarus and Folkman (1984), which distinguished between: 1) a problem-focused strategy, aimed at solving the stress-creating problem and 2) an emotionfocused strategy, aimed at reducing internal distress. Note that whereas these two coping styles are commonly accepted, other coping styles have been recognized; for example help seeking (Lazarus \& Folkman, 1984). Whereas the problem-focused strategy focuses at direct problem solving and at reducing external stressors, the emotion-focused coping style aims to reduce the experienced distress through various mechanisms including reappraisal, selective attention, and avoidance. 
Studies show that emotion-focused coping, particularly avoidance coping, are related to higher levels of PTSD (e.g. Brousse et al., 2011; Bryant \& Harvey, 1995; Chang et al., 2003; Ménard \& Arter, 2014; Mikulincer \& Solomon, 1989), while problem-focused coping is associated with lower levels of PTSD (Mikulincer \& Solomon, 1989). As such, the application of emotion-focused coping seems to be maladaptive, while problem-focused coping appears to be adaptive. However, less is known about of the relation between coping strategies and long-term PTSD trajectories.

\subsection{Aims and objectives}

The current study attempts to fill the abovementioned gaps in knowledge by investigating the relation between combat exposure, LOC, coping-style and long-term trajectories of combatinduced PTSD symptoms in soldiers with and without precedent Combat Stress Reaction (CSR).

We capitalize on a 20 years longitudinal study of Israeli veterans (Solomon \& Mikulincer, 2006). The long-term trajectories of PTSD in this sample; namely those of resilience, delayed onset, recovery, and chronicity, were identified in a data-driven fashion by application of Latent Growth Mixture Modeling (LGMM) in a previously published paper (Karstoft et al., 2013). The current study aims to assess the relation of LOC and coping style to the these long-term PTSD trajectories in addition to the contribution of combat exposure. Specifically, we hypothesized that combat exposure (as measured by participant's subjective perception of life threat and severity of battles), external LOC, and emotion-focused coping would increase the risk of belonging to any of the symptomatic trajectories, while problemfocused coping would reduce the risk of belonging to the symptomatic trajectories. We hypothesize that combat exposure, LOC and coping style act as significant covariates of 
trajectories above and beyond the influence of having a combat stress reaction. Hence, we hypothesize that we will find significant associations between these covariates and symptomatic trajectories in the CSR group as well as the non-CSR group.

\section{Material and methods}

Six hundred and seventy five Israeli male veterans from the 1982 war in Lebanon were assessed 1 (T1), 2 (T2), and 20 (T3) years after the war. Of the total sample, 369 were diagnosed with combat stress reaction (CSR) during the war. These were age, education, military rank, and assignment-matched with a control with no antecedent CSR ( $N=306)$. Age at the first measurement ranged from 18 to 37 years $(M=25.81, S D=4.72$, median $=26)$. Of the total sample at T1, 462 also participated at T2, constituting a $68.4 \%$ participation rate at follow-up. Of those 462 veterans, 296 participated at T3, constituting $64.1 \%$ of those who participated in the first two assessments. Importantly, military records and data collected at T1 revealed no significant differences in socio-demographic and military background, pre-military adjustment, intelligence, or mental and somatic health one year after the war between those who participated at all three time points and those who did not. While the attrition was significantly higher for the CSR group (27.3\%) than for the non-CSR group $(19.2 \%)$ at $\mathrm{T} 2\left(X^{2}(2,666)=14.04, p<.001\right)$ there was no difference in attrition between the two groups at T3 (CSR=24.4\% missing, nonCSR $\left.=29.9 \% ; X^{2}(2,666)=.21, p=.644\right)$. To deal with the effects of attrition, all missing data were handled using the Multiple Imputation (MI) technique. In MI, instead of replacing missing values with one value such as the mean, each missing value is replaced by a set of plausible values that represent the uncertainty about which value to impute (Rubin, 1987). We tested the accuracy of the MI procedure by comparing correlations between outcome measures before and 
after imputation and found no differences. Finally, we ran the LGMM model on the non-imputed data set including only participants with at least two assessments. This model was very similar to the model based on imputed data in trajectory profiles as well as in trajectory prevalence. Thus satisfied with the imputation procedure, analyses were based on the imputed data set to maximize group sizes for the post hoc analysis.

\subsection{Measures}

The PTSD inventory (Solomon, Weisenberg, Schwarzwald, \& Mikulincer, 1987), used to assess PTSD symptomatology, is a 13-item self-report questionnaire based on the DSM-III (APA, 1980) criteria for PTSD (DSM-III was the standard used when the study commenced). The instrument has demonstrated good psychometric properties (Solomon \& Horesh, 2007), and its clinical validity was supported by concurrent SCID-interviews (Solomon, 1993). Also, high testretest reliability over a period of two weeks was found (82.3\% agreement; Solomon, 1993). For the purpose of this study, a total score representing the number of endorsed symptoms was computed. The response scale for each item is dichotomous (yes/no), making the range of the total score 0-13. Cronbach's alpha for the total score was high at all assessments (1983 and 1984: $\alpha=.89,2002: \alpha=.92)$.

Subjective combat exposure was assessed by two questions, assessing whether the war was perceived as life threatening ( $1=$ not at all, 5=very much) and how severe the battles in which the veteran had participated were (1=have not participated in any, 4=hard).

Locus of Control was assessed by the 15-item version of Rotter's internal-external Locus of Control Scale (Rotter, 1966) at T1. Each item is comprised of two sentences; one that represents external LOC and one that represents internal LOC. Participants were asked to select the 
sentence with which they agreed the most. Based on their endorsement, an overall internal/external score was computed. The version of the internal/external LOC scale used in this study was found to have acceptable reliability $(\alpha=0.68)$.

Coping Style was assessed via a 44-item version of the Ways of Coping Checklist (Folkman \& Lazarus, 1980) at T2. Participants were asked to recall a recent stressful event to assess their general soping style in stressful situations. For each item, participants rated the degree to which that item represents a behavior they would apply for handling the stressful event ( $1=$ not used, 4=used a great deal). The Ways of Coping Checklist has been used to assess general coping style previously in samples of war veterans (Solomon, Avitzur, \& Mikulincer, 1990). Factor analysis performed in this sample derived four coping factors (Solomon, 1988): emotion-focused, problem-focused, help-seeking, and active distancing. For the present analysis, we used only the emotion-focused and the problem-focused coping styles. We found acceptable internal consistency for the scale in the present sample with a Cronbach's Alpha of 0.90 for the entire scale, 0.86 for problem-focused coping and 0.69 for emotion-focused coping.

\subsection{Analysis}

All analyses were conducted in Mplus version 7 (Muthen \& Muthen, 2012). We utilized the statistical technique of Latent Growth Mixture Modeling (LGMM) to identify trajectories over time (Muthen, 2004). In combining the statistical methods of Latent Class Analysis (LCA) and Growth Modeling, LGMM expects different subpopulations with unique growth trajectories over time (Jung \& Wickrama, 2008). Hence, this statistical method allows identification of prototypical trajectories in a sample, and is therefore ideal for investigating heterogeneous 
trauma responses (Bonanno \& Mancini, 2012). In order to account for the long time span between assessments (1, 2, and 20 years after the war, respectively), these interval were specifically indexed in the model when estimating the slope. To maintain the original study groups of this sample, we utilized the KNOWNCLASS option in Mplus to divide between the CSR and the non-CSR groups. Following this method, the LGMM trajectories were estimated in one single model, but all parameters were allowed to vary freely across groups.

The LGMM analysis defining the trajectories over time in this study was conducted in a previous study (see Karstoft et al., 2013 for details on analytical approach). For the analysis of interest in this paper, we tested the association of our predictor variables with the PTSD trajectories outside the LGMM model. Simulation studies have suggested that for models with high entropy (>.80), covariate estimation based on most likely class membership is a good alternative to including covariates in the model (Clark \& Muthen). Indeed, our model had very high entropy (.93), and we therefore used the most likely class membership as the outcome variable in a multinomial logistic regression. Prior to this analysis, we estimated correlations between predictor variables and tested for multicollinearity. We found moderate significant correlations between the two combat exposure measures $(\mathrm{r}=.47, \mathrm{p}<.01$, and between problem- and emotion-focused coping $(\mathrm{r}=.41, \mathrm{p}<.01)$ as well as weak correlations between LOC and threatening war life $(\mathrm{r}=-.20$, $\mathrm{p}<.01$, LOC and problem-focused coping $(\mathrm{r}=.-.16, \mathrm{p}<.01)$, and between threatening war life and problem-focused coping $(\mathrm{r}=.09, \mathrm{p}<.05)$. No multicollinearity was found.

\section{Results}


A LGMM model with four trajectories was found to provide the best fit our data (for further details of fit across models please refer to Karstoft et al., 2014). For both the CSR and the nonCSR groups, the trajectories were those of resilience, recovery, delayed onset, and chronicity (see Figure 1). However, CSR and non-CSR group differed in prevalence of the four trajectories (resilience: $\mathrm{CSR}=34.4 \%$, non-CSR $=76.5$; recovery: $\mathrm{CSR}=36.3 \%$, non-CSR $=10.5 \%$; delayed onset: $\mathrm{CSR}=5.4 \%$, non-CSR $=6.9 \%$; chronic: $\mathrm{CSR}=20.9 \%$, non-CSR $=6.2 \%$ ). Finally, initial symptom levels were higher for most trajectories in the CSR group (see Figure 1).

The results of the multinomial logistic regression analysis can be seen in Table 1. Whereas severity of battles did not significantly predict membership of the trajectories in either group, perception of life threat was a significant predictor of membership of all symptomatic trajectories in the CSR group (OR's: chronic $=1.76, p=.007$; recovering $=1.95, p<.001$; delayed $=1.91, p=.030$; for confidence intervals see Table 1). For the non-CSR group, perception of life threat significantly predicted membership of the chronic $(\mathrm{OR}=2.44, p=.003)$ and the recovering $(\mathrm{OR}=2.53, \mathrm{p}<.001)$ trajectories, but not the delayed trajectory. For the CSR group as well as the non-CSR group, a more internal locus of control decreased the likelihood of belonging to the chronic (OR's: CSR=0.87, $p=.036$; non-CSR=.77, $p=.010$ ), and the recovering (OR's: $\mathrm{CSR}=0.84, p=.003$; non-CSR, $p=.010$ ) trajectories compared to the resilient trajectory. Less use of problem-focused coping increased the likelihood of belonging to the chronic trajectory in the CSR group ( $O R=2.12, p=.011)$, while this association only reached borderline significance in the non-CSR group $(O R=3.06, \mathrm{p}=.050)$. For the non-CSR group, less use of problem-focused coping increased the likelihood of belonging to the delayed-onset group $(O R=3.11, p=.025)$. Finally, less used of emotion-focused coping significantly reduced the likelihood of belonging to the chronic trajectory for the CSR group ( $\mathrm{OR}=.28, p<.001)$, while again this relation was only borderline 
significant for the non-CSR group ( $\mathrm{OR}=.27, p=.050$ ). For the non-CSR group, however, less use of emotional coping significantly decreased the likelihood of belonging to the recovering trajectory ( $O R=.34, p=.041)$, and the delayed trajectory $(O R=.28, p=.038)$, while this was not the case for the CSR group.

\section{Discussion}

Building on existing research, the current study utilized four previously identified (Karstoft et al., 2014) longitudinal PTSD-trajectories of resilience, recovery, delay, and chronicity in veterans with and without CSR to investigate the role of subjectively reported combat exposure, LOC, and coping style. We found that the subjective perception of life threat predicted membership of the chronic and recovering trajectories in both groups as well as the delayed trajectory in the CSR group. A more internal LOC lowered the risk of belonging to the recovering and chronic trajectories in both groups. With regards to coping mechanisms, less use of problem-focused coping was associated with higher likelihood of chronic trajectory membership in the CSR group and delayed trajectory membership in the non-CSR group. For emotion-focused coping, chronic trajectory membership in the CSR group was associated with emotional coping, while this was not the case for the non-CSR group. In this group, however, less use of emotional coping was associated with lower risk of belonging to the recovering and delayed trajectories.

The LGMM analysis provided useful information on the importance of CSR in predicting longterm outcome of PTSD. While four similar trajectories (resilience, recovery, delayed onset, and chronicity) were identified in both groups, these trajectories differed across the two groups in terms of prevalence and initial symptom level. More over; more people belonged to the 
symptomatic groups in the CSR group compared to the non-CSR group, and the level of symptoms was generally higher in the CSR group. As expected, CSR were related to more severe symptomatology over time for a larger group of people.

Since combat exposure proved to be a strong predictor of PTSD-trajectories in a previous analysis of this dataset, we included it in the current analysis to assess its prediction of PTSDtrajectories in combination with a different set of predictors, and to control for it when investigating the role of LOC and coping style. For the role of combat exposure we found that whereas severity of battles, contrary to our hypothesis, was not significantly predictive of longterm PTSD outcome, the perception of the war as being life threatening was predictive of membership of the chronic and the recovering trajectories, but not of the delayed trajectory. This was partly in line with our hypotheses, and suggests that exposure to war in itself does not cause symptomatology, but the perception of life threat does.

In an earlier wave of this study, more internal LOC at the first year after the war was found to significantly predict reductions in PTSD symptoms from the first year after the war to the second year after the war in soldiers suffering from CSR (Solomon et al., 1988). We expanded these findings by relating LOC to heterogeneous 20 -year PTSD-trajectories, showing that LOC is a significant predictor of long-term adaptation in this military sample. More specifically, having a more internal LOC decreased the likelihood of belonging to the chronic and the recovering trajectories in the CSR group as well as the non-CSR group. Hence, and in line with our hypothesis, internal control at the time of the event seems to be an important protective factor against both acute and chronic distress. This is much in line with earlier research, which has suggested that internal LOC is not only a protective factor against PTSD, but might even be a resilience-promoting factor (Hoge, Austin, \& Pollack, 2007). Interestingly, however, internal 
LOC did not decrease the likelihood of belonging to the delayed trajectory. This might suggest that internal locus of control is not a strong predictive factor of long-term resilience in the wake of combat exposure. However, since the gap between the second and the third measurement is 18 years, it is not possible to account for all factors that might have influenced the relationship between LOC and PTSD trajectories in that time gap.

In support of our hypotheses, we found that less use of emotional coping as assessed two years after the war was associated with lower risk of chronic trajectory membership in the CSR group, while this association was only borderline significant in the non-CSR group $(p=0.050)$. The decreased risk of chronicity (at least in the CSR group) in subjects who do not use emotional coping suggest that emotional coping is a maladaptive trauma coping mechanism, at least in relation to the development of chronic PTSD symptoms after combat. In the non-CSR group, less use of emotional coping also decreased the likelihood of belonging to the delayed and the recovering trajectories. This is in line with the view that emotional coping is maladaptive when dealing with combat stress, in the short as well as in the long term. However, it is important to note that coping in our study was not assessed until two years after the war. Studies of coping style and PTSD in war veterans (Benotsch, et al., 2000) have found that not only does initial use of emotional coping predict long-term PTSD symptoms; initial PTSD symptoms is also predictive of more use of emotional coping. Hence, over use of emotional coping might be as much as result as a predictor of psychological reactions to trauma. In the current study, we are not able to disentangle the causal relationship between emotional coping and long-term PTSDtrajectories.

For problem-focused coping, a different pattern emerged: in line with our hypothesis, less use of problem-focused coping increased the probability of belonging to the chronic trajectory for the 
CSR group, while this association was only borderline significant in the non-CSR group. Furthermore, in the non-CSR group, less use of problem-focused coping increased the likelihood of belonging to the delayed onset trajectory. These findings suggest that problem-focused coping is an adaptive form of coping in this sample of war veterans, and hence, that non-use of this strategy increases the risk of chronic and/or delayed reactions after combat exposure. For the CSR-group, non-use of problem-focused coping increases the risk of remaining in the chronic trajectory, while for the non-CSR group it increases the risk of developing symptoms with delayed onset. For the chronic and the delayed onset trajectories, the symptomatology endpoint is similar: high levels of symptoms 20 years after the war. Taken together, these findings are in line with earlier findings of emotion focused coping as maladaptive and problem focused coping as adaptive (Brousse et al., 2011; Bryant \& Harvey, 1995; Chang et al., 2003; Mikulincer \& Solomon, 1989). However, as for emotional coping, it should be taken into account that problemfocused coping in this study was not assessed until the second time point, hence questioning if low PTSD-symptomatology is the result of problem-focused coping, or if low PTSDsymptomatology leads to more use of problem-focused coping.

Our findings furthermore suggest that individual ways of coping with trauma and its sequelae plays a significant role not only in the acute PTSD response, but also in long-term PTSD outcomes. Cognitive theories of PTSD assert that emotional coping maintains PTSD through the hindering of adequate emotional processing of the trauma and thereby failure to recover (Ehlers $\&$ Clark, 2000). Our findings lend support to this assumption and further suggest that the use of problem-focused coping might have the reverse effect of protecting against chronic or worsening outcomes in a long-term perspective. 
Certain limitations must be taken into account when interpreting the results of this study. It is based on self-report questionnaires and therefore relies on introspection which might be biased. Furthermore, a very long time passed between the second and the third measurement (18 years). We cannot account for what happened with the participants in those years, and fluctuations in trajectories might have remained undiscovered. Furthermore, a general limitation is that while investigating the influence of particular constructs such as LOC and coping style, it is not possible to measure, account, and control for all other possible variables of interest. Finally, and importantly, coping style was not assessed until the second time point, and must therefore be considered a covariate and not a predictor of trajectory membership.

In spite of these limitations, the study adds important new perspective to the current field of PTSD research: whereas LOC and coping have previously been found to be associated with PTSD, they have, to the best of our knowledge, not been investigated as covariates of long-term heterogeneous trajectories of PTSD-outcome. Our results show that LOC as well as coping style are important in the genesis of PTSD symptoms. Moreover, our findings suggest that coping style plays a role in not only symptoms genesis, but symptom maintenance. These findings are of great clinical importance, in that they suggest that both LOC and ways of coping are important for the long-term posttraumatic adaptation and hence should be addressed using effective treatment. 


\section{References}

Andersen, S.B., Karstoft, K.I., Bertelsen, M. \& Madsen, T. (2014): Latent trajectories of trauma symptoms and resilience: the 3-year longitudinal prospective USPER study of Danish veterans deployed in Afghanistan. The Journal of Clinical Psychiatry, 75 (9), 1001-1008

Benotsch, E. G., Brailey, K., Vasterling, J. J., Uddo, M., Constans, J. I., \& Sutker, P. B. (2000). War Zone stress, personal and environmental resources, and PTSD symptoms in Gulf War Veterans: A longitudinal perspective. Journal of Abnormal Psychology, 109(2), 205-213. doi:10.1037/0021-843X.109.2.205

Bonanno, George A., \& Mancini, Anthony D. (2012). Beyond resilience and PTSD: Mapping the heterogeneity of responses to potential trauma. Psychological Trauma: Theory, Research, Practice, and Policy, 4(1), 74-83.

Bonanno, George A., Mancini, Anthony D., Horton, Jaime L., Powell, Teresa M., LeardMann, Cynthia A., Boyko, Edward J., .. . Smith, Tyler C. (2012). Trajectories of trauma symptoms and resilience in deployed US military service members: Prospective cohort study. The British Journal of Psychiatry, 200(4), 317-323.

Brewin, Chris R., Andrews, Bernice, \& Valentine, John D. (2000). Meta-analysis of risk factors for posttraumatic stress disorder in trauma-exposed adults. Journal of Consulting and Clinical Psychology, 68(5), 748-766.

Brousse, G., Arnaud, B., Roger, J. D., Geneste, J., Bourguet, D., Zaplana, F., . . Jehel, L. (2011). Management of traumatic events: influence of emotion-centered coping strategies on the occurrence of dissociation and post-traumatic stress disorder. Neuropsychiatric disease and treatment, 7, 127-133. doi: 10.2147/NDT.S17130 
Bryant, R. A., \& Harvey, A. G. (1995). Avoidant coping style and post-traumatic stress following motor vehicle accidents. Behaviour Research and Therapy, 33(6), 631-635. doi: 000579679400093 Y [pii]

Casella, Lauraine, \& Motta, Robert W. (1990). Comparison of characteristics of Vietnam veterans with and without Posttraumatic Stress Disorder. Psychological Reports, 67(2), 595-605.

Chang, C. M., Lee, L. C., Connor, K. M., Davidson, J. R., Jeffries, K., \& Lai, T. J. (2003). Posttraumatic distress and coping strategies among rescue workers after an earthquake. Journal of Nervous and Mental Disease, 191(6), 391-398. doi: 10.1097/01.NMD.0000071588.73571.3D [doi]

Clark, S. L., \& Muthen, B. O.. Relating Latent Class Analysis Results to Variables not Included in the Analysis. Mplus webnote: http://www.statmodel.com/download/relatinglca.pdf

Dickstein, B. D., Suvak, M., Litz, B. T., \& Adler, A. B. (2010). Heterogeneity in the course of posttraumatic stress disorder: trajectories of symptomatology. Journal of Traumatic Stress, 23(3), 331-339. doi: 10.1002/jts.20523

Ehlers, A., \& Clark, D. M. (2000). A cognitive model of posttraumatic stress disorder. Behaviour Research and Therapy, 38(4), 319-345. doi: S0005-7967(99)00123-0 [pii]

Folkman, S., \& Lazarus, R. S. (1980). An analysis of coping in a middle-aged community sample. Journal of Health and Social Behavior, 21(3), 219-239.

Hoge, E. A., Austin, E. D., \& Pollack, M. H. (2007). Resilience: research evidence and conceptual considerations for posttraumatic stress disorder. Depression and Anxiety, 24(2), 139-152. doi: 10.1002/da.20175 [doi] 
Jung, Tony, \& Wickrama, K. (2008). An introduction to latent class growth analysis and growth mixture modeling. Social and Personality Psychology Compass, 2(1), 302-317.

Karstoft, K. I., Armour, C., Elklit, A., \& Solomon, Z. (2013). Long-term trajectories of PTSD in veterans: the role of social resources. Journal of Clinical Psychiatry, 74(12):1163-8

Kessler, R. C., Sonnega, A., Bromet, E., Hughes, M., \& Nelson, C. B. (1995). PosttraumaticStress-Disorder in the National Comorbidity Survey. Archives of General Psychiatry, 52(12), 1048-1060.

Lazarus, R. S. , \& Folkman, S. (1984). Stress, Appraisal, and Coping. New York: Springer.

McKeever, Victoria M., McWhirter, Benedict T., \& Huff, Maureen E. (2006). Relationships Between Attribution Style, Child Abuse History, and PTSD Symptom Severity in Vietnam Veterans. Cognitive Therapy and Research, 30(2), 123-133.

Meichenbaum, Donald H., \& Deffenbacher, Jerry L. (1988). Stress Inoculation Training. The Counseling Psychologist, 16(1), 69-90. doi: 10.1177/0011000088161005

Ménard, K. S., \& Arter, M. L. (2014). Stress, coping, alcohol use, and posttraumatic stress disorder among an international sample of police officers: Does gender matter? Police Quarterly, 17(4), 307-327. doi:10.1177/1098611114548097Mikulincer, M., \& Solomon, Z. (1989). Causal attribution, coping strategies, and combat-related post-traumatic stress disorder. European Journal of Personality, 3(4), 269-284.

Muthen, B. O. (2004). Latent variable analysis: Growth mixture modeling and related techniques for longitudinal data. In D. Kaplan (Ed.), Handbook of quantitative methodology for the social sciences. Newbury Park, CA: Sage Publications.

Muthen, B. O., \& Muthen, L. K. (2012). Mplus (version 7). 
Norris, F. H., Friedman, M. J., Watson, P. J., Byrne, C. M., Diaz, E., \& Kaniasty, K. (2002). 60,000 disaster victims speak: Part I. An empirical review of the empirical literature, 1981-2001. Psychiatry, 65(3), 207-239.

Olff, M., Langeland, W., \& Gersons, B. P. (2005). The psychobiology of PTSD: coping with trauma. Psychoneuroendocrinology, 30(10), 974-982. doi: 10.1016/j.psyneuen.2005.04.009

Orcutt, Holly K., Erickson, Darin J., \& Wolfe, Jessica. (2004). The course of PTSD symptoms among Gulf War Veterans: A growth mixture modeling approach. Journal of Traumatic Stress, 17(3), 195-202.

Ozer, E. J., Best, S. R., Lipsey, T. L., \& Weiss, D. S. (2003). Predictors of posttraumatic stress disorder and symptoms in adults: A meta-analysis. Psychological Bulletin, 129(1), 52-73. doi: Doi 10.1037//0033-2909.129.1.52

Rotter, J. B. (1966). Generalized expectancies for internal versus external control of reinforcement. Psychological Monographs, 80(1), 1-28.

Rubin, D. B. (1987). Multiple Imputation for Nonresponse in Surveys New York: John Wiley \& Sons.

Solomon, Z. (1993). Combat stress reaction: The enduring toll of war. New York: Plenum.

Solomon, Z., Avitzur, E., \& Mikulincer, M. (1990). Coping styles and post-war psychopathology among Israeli soldiers. Personality and Individual Differences, 11, 451-456.

Solomon, Z., \& Horesh, D. (2007). Changes in diagnostic criteria of PTSD: Implications from two prospective longitudinal studies. American Journal of Orthopsychiatry, 77(2), 182188. 
Solomon, Z., \& Mikulincer, M. (2006). Trajectories of PTSD: A 20-year longitudinal study. American Journal of Psychiatry, 163(4), 659-666.

Solomon, Z., Mikulincer, M., \& Avitzur, E. (1988). Coping, locus of control, social support, and combat-related posttraumatic stress disorder: A prospective study. Journal of Personality and Social Psychology, 55(2), 279-285.

Solomon, Z., Weisenberg, M., Schwarzwald, J., \& Mikulincer, M. (1987). Posttraumatic stress disorder among frontline soldiers with combat stress reaction: the 1982 Israeli experience. American Journal of Psychiatry, 144(4), 448-454.

Zhang, W., Liu, H., Jiang, X., Wu, D., \& Tian, Y. (2014). A longitudinal study of posttraumatic stress disorder symptoms and its relationship with coping skill and locus of control in adolescents after an earthquake in China. PLoS ONE, 9(2). 